\title{
Quark mass matrices in magnetized orbifold models with localized Fayet-Iliopoulos terms
}

\author{
Hiroyuki Abe, ${ }^{1}$ Tatsuo Kobayashi, ${ }^{2}$ Shintaro Takada, ${ }^{2}$ Shio Tamba, ${ }^{2}$ and Takuya H. Tatsuishi ${ }^{2}$ \\ ${ }^{1}$ Department of Physics, Waseda University, Tokyo 169-8555, Japan \\ ${ }^{2}$ Department of Physics, Hokkaido University, Sapporo 060-0810, Japan
}

(Received 20 July 2018; published 21 November 2018)

\begin{abstract}
We study magnetized orbifold models. We assume the localized Fayet-Iliopoulos terms and the corresponding gauge background. Such terms lead to the strong localization of zero-mode wave functions. In this setup, we compute quark mass matrices.
\end{abstract}

DOI: $10.1103 /$ PhysRevD.98.106017

\section{INTRODUCTION}

Superstring theory is a promising candidate for a unified theory of all the interactions including gravity, quarks, and leptons as well as Higgs fields. In addition to our four-dimensional (4D) spacetime, superstring theory predicts extra six-dimensional space, which should be compact. Thus, extra dimensional models are well motivated.

The standard model is the chiral theory. The torus compactification is one of the simplest compactifications, but the torus compactification leads to a 4D nonchiral lowenergy effective field theory. Thus, the simple torus compactification is not realistic. How to derive a chiral theory is a key point when we start from extra dimensional field theory and superstring theory.

The torus compactification with a magnetic flux is a simple way to derive a $4 \mathrm{D}$ chiral theory from extra dimensional field theory and superstring theory [1-4]. The number of zero modes, i.e., the generation number, is determined by the magnitude of the magnetic flux. Their zero-mode wave functions are quasilocalized around points different from each other in the compact space. Such behavior can lead to suppressed couplings, which would be useful to explain quark and lepton masses and mixing angles. Indeed, in Ref. [5], Yukawa couplings were computed. In addition, higherorder couplings were computed in Ref. [6]. Quark and lepton mass matrices were also discussed. (See, e.g., Refs. [7,8].)

The orbifold compactification with a magnetic flux is also interesting [9]. Orbifolding can project out the adjoint

Published by the American Physical Society under the terms of the Creative Commons Attribution 4.0 International license. Further distribution of this work must maintain attribution to the author(s) and the published article's title, journal citation, and DOI. Funded by SCOAP . matter fields, i.e., open string moduli. ${ }^{1}$ Also, the number of zero modes and their wave functions on orbifolds with magnetic fluxes are different from those on a torus compactification with a magnetic flux $[9,11,12]$. Thus, the orbifold compactification makes model building rich. For example, the realization of quark and lepton masses and mixing angles as well as $C P$ phases was studied [13-18]. However, such a realization is still a challenging issue.

In addition, we can assume localized operators on orbifold fixed points [19-23]. That also makes model building more rich. In Ref. [24], Fayet-Iliopoulos (FI) terms [25], which are localized on the fixed points, were studied on the $T^{2} / Z_{2}$ orbifold compactification (without a magnetic flux). Although the zero-mode profile is flat without localized FI terms, zero modes are localized around orbifold fixed points because of FI terms. This behavior would be interesting from the phenomenological viewpoint.

In this paper, we study the $Z_{2}$ orbifold compactification with magnetic fluxes and localized FI terms. Localized FI terms drastically change the zero-mode profiles from those without FI terms. That would also change the pattern of fermion mass matrices. In this setup, we investigate the realization of quark masses and mixing angles.

This paper is organized as follows. In Sec. II, we explain our setup. In Sec. III, we examine quark masses and mixing angles in our model. Section IV is the conclusion and discussion. In the Appendix, we show wave functions explicitly, which are used in our analysis.

\section{MAGNETIZED ORBIFOLD MODELS WITH LOCALIZED FI TERMS}

In this section, we explain our setup and zero-mode profiles. We implicitly assume that our models are supersymmetric. For example, when the number of compact

\footnotetext{
${ }^{1}$ This aspect corresponds to the $T$-dual picture of intersecting D-brane models on orbifolds [10].
} 
dimensions is six (four), the compact space is the tensor product of three (two) two-dimensional (2D) spaces. We assume that magnetic fluxes in three (two) 2D compact spaces are set such that $4 \mathrm{D} N=1$ supersymmetry is unbroken. However, the flavor structure, i.e., the difference between fermion flavors, originates from one of the 2D compact spaces. Hence, we concentrate on the 2D compact spaces such as $T^{2}$ and $T^{2} / Z_{2}$, because this part is important to realize Yukawa matrices consistent with observations. Then, we study the zero-mode wave functions and Yukawa couplings on the $2 \mathrm{D}$ space.

\section{A. Torus compactification with a magnetic flux}

Here, we briefly review the torus model with magnetic fluxes [5]. We use the complex coordinate $z=x+\tau y$ instead of the real coordinates $(x, y)$, where $\tau$ is a complex structure modulus. By use of the complex coordinate, the metric is written by $d s^{2}=g_{\alpha \beta} d z^{\alpha} d \bar{z}^{\beta}$,

$$
g_{\alpha \beta}=\left(\begin{array}{ll}
g_{z z} & g_{z \bar{z}} \\
g_{\bar{z} z} & g_{\bar{z} \bar{z}}
\end{array}\right)=(2 \pi R)^{2}\left(\begin{array}{cc}
0 & \frac{1}{2} \\
\frac{1}{2} & 0
\end{array}\right) \text {. }
$$

To realize the $T^{2}$, we identify $z \sim z+1$ and $z \sim z+\tau$.

We consider the $\mathrm{U}(1)$ theory with the following magnetic flux:

$$
F=i \frac{\pi M}{\operatorname{Im} \tau}(d z \wedge d \bar{z})
$$

where $M$ must be quantized to be an integer. This flux can be obtained by the following vector potential:

$$
A(z)=\frac{\pi M}{\operatorname{Im} \tau} \operatorname{Im}(\bar{z} d z),
$$

in a certain gauge.

Here, we study the spinor field $\psi(z, \bar{z})$ with $\mathrm{U}(1)$ charge $q$ on $T^{2}$, which has two components:

$$
\psi(z, \bar{z})=\left(\begin{array}{c}
\psi_{+} \\
\psi_{-}
\end{array}\right) .
$$

Then, we examine the zero-mode equation

$$
i \not D \psi=0,
$$

which can be written in components

$$
D \psi_{+}=0, \quad D^{\dagger} \psi_{-}=0,
$$

where

$$
D^{\dagger} \equiv \partial-q \frac{\pi M}{2 \operatorname{Im} \tau} \bar{z}, \quad D \equiv \bar{\partial}+q \frac{\pi M}{2 \operatorname{Im} \tau} z
$$

Also, they must satisfy the following boundary condition:

$\psi_{ \pm}(z+1)=e^{i q \phi_{1}(z)} \psi_{ \pm}(z)=\exp \left\{i \frac{\pi q M}{\operatorname{Im} \tau} \operatorname{Im} z\right\} \psi_{ \pm}(z)$

$\psi_{ \pm}(z+\tau)=e^{i q \phi_{2}(z)} \psi_{ \pm}(z)=\exp \left\{i \frac{\pi q M}{\operatorname{Im} \tau} \operatorname{Im} \bar{\tau} z\right\} \psi_{ \pm}(z)$

Either $\psi_{+}$or $\psi_{-}$has zero-mode solutions exclusively when $q M \neq 0$. That is, for $q M>0(q M<0), \psi_{+}\left(\psi_{-}\right)$has $|q M|$ solutions, while $\psi_{-}\left(\psi_{+}\right)$has no zero modes. The number $|q M|$ would correspond to the generation number. Their zero-mode profiles for $q M>0$ are given by

$$
\psi^{j, q M}(z)=\mathcal{N} e^{i \pi q M z^{\frac{\mathrm{Im} z}{\mathrm{Im} \tau}}} \cdot \vartheta\left[\begin{array}{c}
\frac{j}{q M} \\
0
\end{array}\right](q M z, q M \tau),
$$

with $j=0,1, \ldots,(|q M|-1)$, where $\vartheta$ denotes the Jacobi theta function

$$
\vartheta\left[\begin{array}{l}
a \\
b
\end{array}\right](\nu, \tau)=\sum_{l \in \mathbf{Z}} e^{\pi i(a+l)^{2} \tau} e^{2 \pi i(a+l)(\nu+b)} .
$$

Here, $\mathcal{N}$ denotes the normalization factor given by

$$
\mathcal{N}=\left(\frac{2 \operatorname{Im} \tau q M}{\mathcal{A}^{2}}\right)^{1 / 4},
$$

with $\mathcal{A}=4 \pi^{2} R^{2} \operatorname{Im} \tau$. The scalar fields have the same wave functions as the spinor fields.

\section{B. $T^{2} / Z_{2}$ orbifold}

The $T^{2} / Z_{2}$ orbifold can be constructed from $T^{2}$ by identifying $z \sim-z$ through the $Z_{2}$ twist. For simplicity, we set $\tau=i \operatorname{Im} \tau$. There are four fixed points on $T^{2} / Z_{2}$, i.e.,

$$
z_{I}=0, \frac{1}{2}, \frac{i}{2} \operatorname{Im} \tau, \frac{1}{2}+\frac{i}{2} \operatorname{Im} \tau .
$$

By the $Z_{2}$ twist $z \rightarrow-z$, the zero modes can be classified into the $Z_{2}$ even and odd modes. The zero modes $\psi^{j, q M}$ satisfy the following relation:

$$
\psi^{j, q M}(-z)=\psi^{q M-j, q M}(z) .
$$

Note that $\psi^{0, q M}(z)$ is invariant under the $Z_{2}$ twist, and $\psi^{q M / 2, q M}(z)$ is also invariant under the $Z_{2}$ twist when $q M$ is even. Thus, the $Z_{2}$ even modes are

$$
\Theta_{+}^{j, q M}(z)=\frac{1}{\sqrt{2}}\left(\psi^{j, q M}(z)+\psi^{q M-j, q M}(z)\right),
$$


TABLE I. The numbers of $Z_{2}$ even and odd zero modes.

\begin{tabular}{lcc}
\hline \hline$q M$ & $2 n$ & $2 n+1$ \\
\hline$Z_{2}$ even & $n+1$ & $n+1$ \\
$Z_{2}$ odd & $n-1$ & $n$ \\
\hline \hline
\end{tabular}

for $j \neq 0, q M / 2$, in addition to

$$
\begin{aligned}
\Theta_{+}^{0, q M}(z) & =\psi^{0, q M}(z), \\
\Theta_{+}^{q M / 2, q M}(z) & =\psi^{q M / 2, q M}(z),
\end{aligned}
$$

only if $q M$ is even. Similarly, the $Z_{2}$ odd modes are obtained by

$$
\Theta_{-}^{j, q M}(z)=\frac{1}{\sqrt{2}}\left(\psi^{j, q M}(z)-\psi^{q M-j, q M}(z)\right) .
$$

The numbers of zero modes are shown in Table I. The three generations can be obtained as $Z_{2}$ even (odd) modes for $q M=4,5(q M=7,8)$ [13]. When we include nonvanishing Wilson lines, the numbers of $Z_{2}$ even and odd zero modes change [11]. Here, we restrict ourselves to vanishing Wilson lines.

\section{Localized FI terms without a bulk magnetic flux}

Here, we review briefly the FI terms localized on the orbifold fixed points with no bulk magnetic flux [24]. The localized FI terms such as

$$
\xi=\sum_{I=\mathrm{fp}}\left(\xi_{I}+\xi^{\prime \prime}(\partial \bar{\partial})\right) \delta^{2}\left(z-z_{I}\right)
$$

were studied in Ref. [24], where these FI terms were induced by radiative corrections due to bulk and brane modes. ${ }^{2}$ Here, fp means that the summation is taken over the fixed points. Also, it was shown that, under the condition of unbroken supersymmetry, the localized FI terms correspond to the flux $F^{(\xi)}=\xi$, which is obtained by the vector potential $A^{(\xi)}$. Then, the solution of the zeromode equation

$$
\left(\bar{\partial}+q A^{(\xi)}\right) \psi_{+}=0
$$

was studied. Without $A^{(\xi)}$, the zero-mode profile is constant. However, with nonvanishing $A^{(\xi)}$, zero-mode profiles are localized around fixed points, depending on $q \xi_{I}$. Thus, the localized FI terms lead to a strong localization of zeromode wave functions. Explicit solutions for the above zeromode equations were shown in Ref. [24]:

\footnotetext{
${ }^{2}$ Similarly, the FI terms are generated by radiative corrections on the $S^{1} / Z_{2}$ orbifold, and wave function profiles are strongly affected by FI terms to be (quasi)localized. See, e.g., Refs. [26-28].
}

$$
\begin{aligned}
\phi^{(q \xi)}= & \prod_{I=\mathrm{fp}}\left|\vartheta_{1}\left(z-z_{I} \mid \tau\right)\right|^{q \xi_{I} /(2 \pi)} \\
& \times \exp \left[q \xi^{\prime \prime} / R^{2} \delta^{2}\left(z-z_{I}\right)+\cdots\right] .
\end{aligned}
$$

Their wave functions can be singular at the fixed points, which correspond to $q \xi_{I}<0$. A certain regularization was also shown in Ref. [24]. In this paper, we will assume the same form of the gauge background $A^{(\xi)}$. In the model without the bulk flux, $\xi_{I}$ must satisfy [24]

$$
\sum_{I} \xi_{I}=0
$$

That implies that at most three fixed points among the four can have $q \xi_{I}<0$, while the other has $q \xi_{I}>0$. That is, the wave function $\phi^{(q \xi)}$ can be singular at three fixed points at most and be delocalized at the other fixed point. In the model with a nonvanishing bulk flux, Eq. (22) is not satisfied, i.e., $\sum_{I} \xi_{I} \neq 0$. In such a model, the wave function $\phi^{\left(q^{\xi}\right)}$ can be singular at all of the fixed points at most.

\section{Magnetized orbifold with localized FI terms}

Here, we explain our setup. We consider the $T^{2} / Z_{2}$ orbifold compactification with a bulk magnetic flux and FI terms localized on the fixed points. In other words, we assume the vector potential $A^{(\xi)}$, which corresponds effectively to the localized FI terms in the previous section as Ref. [24]. Then, the zero-mode equation, e.g., for $\psi_{+}$, is written by

$$
\left(\bar{\partial}+q \frac{\pi M}{2 \operatorname{Im} \tau} z+q A^{(\xi)}\right) \psi_{+}=0 .
$$

When $A^{(\xi)}=0$, the solutions on the orbifold are obtained as $\Theta_{ \pm}^{j, q M}$. On the other hand, when $q M=0$, the solution is $\phi^{(q \xi)}$. Then, the solutions of the above zero-mode equation can be written by $\Theta_{ \pm}^{j, q M} \phi^{(q \xi)}$. However, $\phi^{(q \xi)}$ has a singular behavior on the fixed points, depending on the sign of $q \xi_{I}$. We need some regularization to obtain finite results.

For example, the Yukawa couplings can be computed by the overlap integral of the wave functions such as

$$
Y_{j k \ell}=g \int d^{2} z\left(\Theta_{ \pm}^{j, q M} \phi^{(q \xi)}\right)\left(\Theta_{ \pm}^{k, q^{\prime} M^{\prime}} \phi^{\left(q^{\prime} \xi\right)}\right)\left(\Theta_{ \pm}^{\ell, q^{\prime \prime} M^{\prime \prime}} \phi^{\left(q^{\prime \prime} \xi\right)}\right)
$$

where $g$ is a coupling in higher-dimensional field theory. When this coupling is $\mathrm{U}(1)$ invariant, we have $\left(q+q^{\prime}+q^{\prime \prime}\right) \xi_{I}=0$. That leads to $\phi^{(q \xi)} \phi^{\left(q^{\prime}(\xi)\right.} \phi^{\left(q^{\prime \prime}\right)}=1$ because of the solution from (21). Then, the Yukawa couplings can be written by 


$$
Y_{j k \ell}=g \int d^{2} z \Theta_{ \pm}^{j, q M} \Theta_{ \pm}^{k, q^{\prime} M^{\prime}} \Theta_{ \pm}^{\ell, q^{\prime \prime} M^{\prime \prime}}
$$

This form is the same as the Yukawa coupling on the orbifold without localized FI terms. The computation can be extended to higher-order couplings. This is one of our results in this paper. That is, even if there are localized FI terms on the orbifold, the U(1)-invariant Yukawa couplings and higher-order couplings are the same forms as those on the orbifold without localized FI terms.

In the above model, phenomenological aspects are the same as those without localized FI terms [13-18]. Different aspects would be obtained if $q+q^{\prime}+q^{\prime \prime} \neq 0$. Thus, we assume here that $\mathrm{U}(1)$ is anomalous and the above Yukawa couplings $Y_{j k \ell}$, i.e., Eq. (24), are induced by nonperturbative effects for $q+q^{\prime}+q^{\prime \prime} \neq 0$. $^{3}$ Furthermore, we assume $\left(q+q^{\prime}+q^{\prime \prime}\right) \xi_{I}<0$ for all of the fixed points to have a rich structure.

In order to derive a finite result on $Y_{j k \ell}$, we need some regularization of $\phi^{\left(q^{\xi}\right)}{ }^{4}$ In any regularization, $\phi^{(q \xi)}$ would have a huge value around the fixed points, while its value in the bulk except fixed points is suppressed very much compared with a huge value at fixed points. Here, instead of using an explicit regularization, we make an ansatz such that the Yukawa couplings can be approximately computed by wave functions on fixed points:

$$
Y_{j k \ell}=\sum_{I=\mathrm{fp}} c_{I} \Theta_{ \pm}^{j, q M}\left(z_{I}\right) \Theta_{ \pm}^{k, q^{\prime} M^{\prime}}\left(z_{I}\right) \Theta_{ \pm}^{\ell, q^{\prime \prime} M^{\prime \prime}}\left(z_{I}\right)
$$

Here, $c_{I}$ would depend on our regularization scheme and the parameters $q M, q^{\prime} M^{\prime}, q^{\prime \prime} M^{\prime \prime}$, and $\xi_{I}$. We use $c_{I}$ in order to parametrize our ignorance.

Note here that $\Theta_{-}^{j, q M}(z=0)=0$ because of $\Theta_{-}^{j, q M}(z)=$ $-\Theta_{-}^{j, q M}(z)$. Similarly, we can show that wave functions of $Z_{2}$ odd modes vanish at all of the fixed points except the fixed point $z_{I}=1 / 2+i \operatorname{Im} \tau / 2$ by use of the boundary conditions (8) and (9), i.e.,

$$
\Theta_{-}^{j, q M}\left(z=z_{I}\right)=0 .
$$

Also, at the fixed point $z_{I}=1 / 2+i \operatorname{Im} \tau / 2$, the wave function vanishes when $\tau=i \operatorname{Im} \tau$ and $M=$ even. Thus, we obtain trivial results for the $Z_{2}$ odd modes in most of the models. Hence, we concentrate on the $Z_{2}$ even modes.

When one of three fields, say, $\Theta_{+}^{\ell, q^{\prime \prime} M^{\prime \prime}}\left(z_{I}\right)$, corresponds to the Higgs field in the above Yukawa coupling, and it develops its vacuum expectation value $v$, fermion masses are obtained as

\footnotetext{
${ }^{3}$ It may be a challenging issue to induce Yukawa couplings of $\mathcal{O}(1)$ for top quarks by nonperturbative effects. In order to study this issue, we have to evaluate nonperturbative effects explicitly. We postpone this issue until future work, but we just assume the form of $Y_{j k \ell}$ shown in Eq. (24).

${ }^{4}$ See, for an explicit form of regularization, Ref. [24].
}

$$
m_{j k}=v \sum_{I=\mathrm{fp}} c_{I} \Theta_{+}^{j, q M}\left(z_{I}\right) \Theta_{+}^{k, q^{\prime} M^{\prime}}\left(z_{I}\right) \Theta_{+}^{\ell, q^{\prime \prime} M^{\prime \prime}}\left(z_{I}\right) .
$$

Many models lead to multiple Higgs fields. (See, e.g., $[13,29]$.) The standard-model Higgs field would correspond to their linear combination, and the other would gain mass terms at some stage. In such multi-Higgs models, fermion masses could be written by

$m_{j k}=\sum_{I=\mathrm{fp}} c_{I} \Theta_{+}^{j, q M}\left(z_{I}\right) \Theta_{+}^{k, q^{\prime} M^{\prime}}\left(z_{I}\right)\left(\sum_{\ell} v^{\ell} \Theta_{+}^{\ell, q^{\prime \prime} M^{\prime \prime}}\left(z_{I}\right)\right)$.

However, these masses can be parametrized as

$$
m_{j k}=\sum_{I=\mathrm{fp}} m_{I} \Theta_{+}^{j, q M}\left(z_{I}\right) \Theta_{+}^{k, q^{\prime} M^{\prime}}\left(z_{I}\right)
$$

Here, four parameters $m_{I}$ include our ignorance in $c_{I}$ and details in the Higgs sector. ${ }^{5}$ In the next section, we use this ansatz to study quark masses and mixing angles. Note that if only one of $m_{I}$ is nonvanishing and the other vanishes, the mass matrix $m_{i j}$ has rank 1 . Thus, two or more $m_{I}$ 's must be nonvanishing.

\section{QUARK MASS MATRICES}

Here we study quark masses and mixing angles by using ansatz (30) in the previous section. Recall that, for simplicity, we set $\tau$ to be pure imaginary, i.e., $\tau=i \operatorname{Im} \tau$. Here we treat $\operatorname{Im} \tau$ as a free parameter, although we need a proper modulus stabilization to realize realistic results. The number of $Z_{2}$ even zero modes is equal to three only if $q M=4$ and 5 . Their wave functions are explicitly shown in the Appendix.

We do not construct a model explicitly. However, we assume that the quark doublets and the up sector of righthanded quarks correspond to $q M=4$, and the down sector of right-handed quarks corresponds to $q M=5$. We assign the first, second, and third families to $\Theta_{+}^{2, q M}, \Theta_{+}^{1, q M}$, and $\Theta_{+}^{0, q M}$, respectively. We also introduce the up sector and down sector of Higgs fields, which are independent of each other. Then, following the ansatz (30) and wave functions shown in the Appendix, the up-sector quark mass matrix can be written

$$
M^{(u)}=M_{0,0}^{(u)}+M_{1 / 2,0}^{(u)}+M_{0,1 / 2}^{(u)}+M_{1 / 2,1 / 2}^{(u)},
$$

where

\footnotetext{
${ }^{5}$ A similar mass matrix is obtained for multiple Higgs generations from localized $\mu$ terms at the fixed points on magnetized orbifolds [23].
} 


$$
\begin{gathered}
M_{0,0}^{(u)}=m_{0,0}^{(u)}\left(\begin{array}{ccc}
4 e^{-2 \tau^{\prime}} & 2 \sqrt{2} e^{-5 \tau^{\prime} / 4} & 2 e^{-\tau^{\prime}} \\
2 \sqrt{2} e^{-5 \tau^{\prime} / 4} & 2 e^{-\tau^{\prime} / 2} & \sqrt{2} e^{-\tau^{\prime} / 4} \\
2 e^{-\tau^{\prime}} & \sqrt{2} e^{-\tau^{\prime} / 4} & 1
\end{array}\right), \\
M_{1 / 2,0}^{(u)}=m_{0,0}^{(u)}\left(\begin{array}{ccc}
4 e^{-2 \tau^{\prime}} & -2 \sqrt{2} e^{-5 \tau^{\prime} / 4} & 2 e^{-\tau^{\prime}} \\
-2 \sqrt{2} e^{-5 \tau^{\prime} / 4} & 2 e^{-\tau^{\prime} / 2} & -\sqrt{2} e^{-\tau^{\prime} / 4} \\
2 e^{-\tau^{\prime}} & -\sqrt{2} e^{-\tau^{\prime} / 4} & 1
\end{array}\right), \\
M_{0.1 / 2}^{(u)}=m_{0,1 / 2}^{(u)}\left(\begin{array}{ccc}
1 & \sqrt{2} e^{-\tau^{\prime} / 4} & 2 e^{-\tau^{\prime}} \\
\sqrt{2} e^{-\tau^{\prime} / 4} & 2 e^{-\tau^{\prime} / 2} & 2 \sqrt{2} e^{-5 \tau^{\prime} / 4} \\
2 e^{-\tau^{\prime}} & 2 \sqrt{2} e^{-5 \tau^{\prime} / 4} & 4 e^{-2 \tau^{\prime}}
\end{array}\right), \\
M_{1 / 2,1 / 2}^{(u)}=m_{1 / 2,1 / 2}^{(u)}\left(\begin{array}{ccc}
1 & -\sqrt{2} e^{-\tau^{\prime} / 4} & 2 e^{-\tau^{\prime}} \\
-\sqrt{2} e^{-\tau^{\prime} / 4} & 2 e^{-\tau^{\prime} / 2} & -2 \sqrt{2} e^{-5 \tau^{\prime} / 4} \\
2 e^{-\tau^{\prime}} & -2 \sqrt{2} e^{-5 \tau^{\prime} / 4} & 4 e^{-2 \tau^{\prime}}
\end{array}\right),
\end{gathered}
$$

up to the normalization factor $\mathcal{N}$. Here we define $\tau^{\prime}=\pi \operatorname{Im} \tau$. Similarly, the down-sector quark mass matrix is written by

$$
M^{(d)}=M_{0,0}^{(d)}+M_{1 / 2,0}^{(d)}+M_{0,1 / 2}^{(d)}+M_{1 / 2,1 / 2}^{(d)}
$$

where

$$
\begin{gathered}
M_{0,0}^{(d)}=m_{0,0}^{(d)}\left(\begin{array}{ccc}
2 \sqrt{2} e^{-9 \tau^{\prime} / 5} & 2 \sqrt{2} e^{-6 \tau^{\prime} / 5} & 2 e^{-\tau^{\prime}} \\
2 e^{-21 \tau^{\prime} / 20} & 2 e^{-9 \tau^{\prime} / 20} & \sqrt{2} e^{-\tau^{\prime} / 4} \\
\sqrt{2} e^{-4 \tau^{\prime} / 5} & \sqrt{2} e^{-\tau^{\prime} / 5} & 1
\end{array}\right), \\
M_{1 / 2,0}^{(d)}=m_{1 / 2,0}^{(d)}\left(\begin{array}{ccc}
2 \sqrt{2} e^{-9 \tau^{\prime} / 5} & -2 \sqrt{2} e^{-6 \tau^{\prime} / 5} & 2 e^{-\tau^{\prime}} \\
-2 e^{-21 \tau^{\prime} / 20} & 2 e^{-9 \tau^{\prime} / 20} & -\sqrt{2} e^{-\tau^{\prime} / 4} \\
\sqrt{2} e^{-4 \tau^{\prime} / 5} & -\sqrt{2} e^{-\tau^{\prime} / 5} & 1
\end{array}\right), \\
M_{0,1 / 2}^{(d)}=m_{0,1 / 2}^{(d)}\left(\begin{array}{ccc}
\sqrt{2} e^{-\tau^{\prime} / 20} & \sqrt{2} e^{-9 \tau^{\prime} / 20} & 2 e^{-5 \tau^{\prime} / 4} \\
2 e^{-6 \tau^{\prime} / 20} & 2 e^{-14 \tau^{\prime} / 20} & 2 \sqrt{2} e^{-3 \tau^{\prime} / 2} \\
2 \sqrt{2} e^{-21 \tau^{\prime} / 20} & 2 \sqrt{2} e^{-29 \tau^{\prime} / 20} & 4 e^{-9 \tau^{\prime} / 4}
\end{array}\right),
\end{gathered}
$$

up to the normalization factor $\mathcal{N}$. In addition, we have $M_{1 / 2,1 / 2}^{(d)}=0 \times m_{1 / 2,1 / 2}^{(d)}$ for $\operatorname{Re} \tau=0$. For $\operatorname{Re} \tau \neq 0$, all the entries of the matrix $M_{1 / 2,1 / 2}^{(d)}$ are nonvanishing, and their absolute values are similar to $M_{0,1 / 2}^{(d)}$.

We have nine parameters, $m_{0,0}^{(u, d)}, m_{1 / 2,0}^{(u, d)}, m_{0,1 / 2}^{(u, d)}, m_{1 / 2,1 / 2}^{(u, d)}$, and $\operatorname{Im} \tau$, and this number of free parameters is enough to fit them to experimental data of six quark masses and three mixing angles. When we include nonvanishing $\operatorname{Re} \tau$, we can also fit the $C P$ phase. Thus, we do not examine the detailed fitting, but we study the order estimation. Note that $M_{0,0}^{(u)}$ $\left(M_{0,1 / 2}^{(u)}\right)$ is very similar to $M_{1 / 2,0}^{(u)}\left(M_{1 / 2,1 / 2}^{(u)}\right)$ and $M_{0,0}^{(d)}$
$\left(M_{0,1 / 2}^{(d)}\right)$ is very similar to $M_{1 / 2,0}^{(d)}\left(M_{1 / 2,1 / 2}^{(d)}\right) .{ }^{6}$ For a simple estimation, we consider the parameter region

$$
m^{(u)} \sim m_{0,0}^{(u)}+m_{1 / 2,0}^{(u)} \sim m_{0,0}^{(u)}-m_{1 / 2,0}^{(u)},
$$

$$
\begin{aligned}
\rho^{(u)} m^{(u)} & \sim m_{0,1 / 2}^{(u)}+m_{1 / 2,1 / 2}^{(u)} \sim m_{0,1 / 2}^{(u)}-m_{1 / 2,1 / 2}^{(u)}, \\
m^{(d)} & \sim m_{0,0}^{(d)}+m_{1 / 2,0}^{(d)} \sim m_{0,0}^{(d)}-m_{1 / 2,0}^{(d)}, \\
\rho^{(d)} m^{(d)} & \sim m_{0,1 / 2}^{(d)} .
\end{aligned}
$$

Then, the quark mass matrices can be written by

\footnotetext{
${ }^{6}$ When $\operatorname{Re} \tau \neq 0, M_{0,1 / 2}^{(d)}$ is similar to $M_{1 / 2,1 / 2}^{(d)}$.
} 


$$
\begin{gathered}
\frac{M^{(u)}}{m^{(u)}} \sim\left(\begin{array}{ccc}
4 e^{-2 \tau^{\prime}}+\rho^{(u)} & \sqrt{2}\left(2 e^{-5 \tau^{\prime} / 4}+\rho^{(u)} e^{-\tau^{\prime} / 4}\right) & 2 e^{-\tau^{\prime}}\left(1+\rho^{(u)}\right) \\
\sqrt{2}\left(2 e^{-5 \tau^{\prime} / 4}+\rho^{(u)} e^{-\tau^{\prime} / 4}\right) & 2 e^{-\tau^{\prime} / 2}\left(1+\rho^{(u)}\right) & \sqrt{2}\left(e^{-\tau^{\prime} / 4}+2 \rho^{(u)} e^{-5 \tau^{\prime} / 4}\right) \\
2 e^{-\tau^{\prime}}\left(1+\rho^{(u)}\right) & \sqrt{2}\left(e^{-\tau^{\prime} / 4}+2 \rho^{(u)} e^{-5 \tau^{\prime} / 4}\right) & 1+4 \rho^{(u)} e^{-2 \tau^{\prime}}
\end{array}\right), \\
\frac{M^{(d)}}{m^{(d)}} \sim\left(\begin{array}{ccc}
\sqrt{2}\left(2 e^{-9 \tau^{\prime} / 5}+\rho^{(d)} e^{-\tau^{\prime} / 20}\right) & \sqrt{2}\left(2 e^{-6 \tau^{\prime} / 5}+\rho^{(d)} e^{-9 \tau^{\prime} / 20}\right) & 2\left(e^{-\tau^{\prime}}+\rho^{(d)} e^{-5 \tau^{\prime} / 4}\right) \\
2\left(e^{-21 \tau^{\prime} / 20}+\rho^{(d)} e^{-6 \tau^{\prime} / 20}\right) & 2\left(e^{-9 \tau^{\prime} / 20}+\rho^{(d)} e^{-14 \tau^{\prime} / 20}\right) & \sqrt{2}\left(e^{-\tau^{\prime} / 4}+2 \rho^{(d)} e^{-3 \tau^{\prime} / 2}\right) \\
\sqrt{2}\left(e^{-4 \tau^{\prime} / 5}+2 \rho^{(d)} e^{-21 \tau^{\prime} / 20}\right) & \sqrt{2}\left(e^{-\tau^{\prime} / 5}+2 \rho^{(d)} e^{-29 \tau^{\prime} / 20}\right) & 1+4 \rho^{(d)} e^{-9 \tau^{\prime} / 4}
\end{array}\right) .
\end{gathered}
$$

It is very straightforward to realize the top and bottom quark masses. Hence, we try to fit our three parameters $\rho^{(u),(d)}$ and $\tau^{\prime}$ to mass ratios $m_{c} / m_{t}, m_{u} / m_{t}, m_{s} / m_{b}$, and $m_{d} / m_{b}$ and mixing angles, seven observables. The experimental values of the Cabibbo-Kobayashi-Maskawa matrix are

$$
\left|V_{\mathrm{CKM}}\right|=\left(\begin{array}{ccc}
0.97 & 0.23 & 0.0035 \\
0.23 & 0.97 & 0.041 \\
0.0087 & 0.040 & 1.0
\end{array}\right)
$$

For example, at $1 \mathrm{TeV}$, the ratios of running masses are obtained as $[30,31]$

$$
\begin{array}{ll}
\frac{m_{c}}{m_{t}}=3.5 \times 10^{-3}, & \frac{m_{u}}{m_{t}}=7.3 \times 10^{-6}, \\
\frac{m_{s}}{m_{b}}=1.9 \times 10^{-2}, & \frac{m_{d}}{m_{b}}=1.0 \times 10^{-3} .
\end{array}
$$

We concentrate on the parameter region $\left|\rho^{(u, d)}\right| \ll 1$. First, we study the mass matrices of the second and third generations, which are written for $\left|\rho^{(u, d)}\right| \ll 1$ as, respectively,

$$
\begin{aligned}
& \frac{M^{(u)}}{m^{(u)}} \sim\left(\begin{array}{cc}
2 e^{-\tau^{\prime} / 2} & \sqrt{2} e^{-\tau^{\prime} / 4} \\
\sqrt{2} e^{-\tau^{\prime} / 4} & 1
\end{array}\right), \\
& \frac{M^{(d)}}{m^{(d)}} \sim\left(\begin{array}{cc}
2 e^{-9 \tau^{\prime} / 20} & \sqrt{2} e^{-\tau^{\prime} / 4} \\
\sqrt{2} e^{-\tau^{\prime} / 5} & 1
\end{array}\right) .
\end{aligned}
$$

These mass matrices include only one free parameter $\tau^{\prime}$ and are very predictable. For example, we take $\sqrt{2} e^{-\tau^{\prime} / 4}=0.08$. Then, we obtain

$$
\frac{m_{c}}{m_{t}}=6.4 \times 10^{-3}, \quad \frac{m_{s}}{m_{b}}=1.1 \times 10^{-2}, \quad V_{c b}=0.08 .
$$

These orders are consistent with experimental values.

Next, we examine the other mixing angles and the mass ratios $m_{u} / m_{t}$ and $m_{d} / m_{b}$. For example, when we take $\rho^{(u)} \sim 7.3 \times 10^{-3}$, we can realize the experimental values $m_{u} / m_{t}$. Also, when we take $\rho^{(d)}=\mathcal{O}(0.1)$, we can realize the experimental order of $V_{u s}$. However, for this value of $\rho^{(d)}$, we have a large ratio, $m_{d} / m_{b}=\mathcal{O}(0.1)$. On the other hand, when we take $\rho^{(d)}=\mathcal{O}(0.001)$, we can realize the experimental value $m_{d} / m_{b}$, but we have a small value $V_{u s}=\mathcal{O}(0.001)$. Thus, we can realize most of the experimental values by the simple parameter region (40), although there is tension between $m_{d} / m_{b}$ and $V_{u s}$. However, by tuning $m_{0,1 / 2}^{(d)}$ and $m_{1 / 2,1 / 2}^{(d)}$ as well as $\operatorname{Re} \tau$, we can realize both $m_{d} / m_{b}$ and $V_{u s}$.

\section{CONCLUSION}

We have studied magnetized orbifold models. We have assumed the FI terms localized at fixed points and the corresponding gauge background. Such terms lead to a strong localization of zero-mode wave functions. We have computed quark mass matrices by parameterizing detail of models and our ignorance. The forms of quark mass matrices are quite simple, but we can fit the experimental data $m_{c} / m_{t}, m_{s} / m_{b}$, and $V_{c b}$ roughly by just one parameter, $\tau^{\prime}$. We can also realize $m_{u} / m_{t}$. However, there is tension between $m_{d} / m_{b}$ and $V_{u s}$ in the simple parameter region, although we can tune parameters to realize both $m_{d} / m_{b}$ and $V_{u s}$.

Similarly, we can discuss the lepton sector. For the mass matrix of charged leptons, the analysis is similar and straightforward. We can realize charged lepton masses. For the neutrino masses and mixing angles, it is an important issue how to derive neutrino masses. For example, right-handed Majorana neutrino masses can be generated on magnetized orbifold models by D-brane instanton effects [15]. It would be important to study such D-brane instanton effects under the background corresponding the localized FI terms.

In this paper, we have concentrated on the $T^{2} / Z_{2}$ orbifold without discrete Wilson lines. It would be interesting to extend our analysis to the $T^{2} / Z_{2}$ orbifold with discrete Wilson lines and other orbifolds with discrete Wilson lines [11]. The numbers of fixed points on the other orbifolds are different from one of the $T^{2} / Z_{2}$ orbifold, and their fixed point structures are different. For example, the $T^{2} / Z_{3}$ orbifold has three fixed points, and the number of free parameters corresponding to $c_{I}$ and $m_{I}$ is three. Hence, it would be intriguing to study the $T^{2} / Z_{3}$ orbifold. We would study elsewhere. 


\section{ACKNOWLEDGMENTS}

The authors thank M. Ishida for useful discussions. H. A. is supported in part by JSPS KAKENHI Grant No. JP16K05330. T. K. is supported in part by MEXT KAKENHI Grant No. JP17H05395. T. H. T. is supported in part by a Grant-in-Aid for JSPS Research Fellow (No. 18J11233).

\section{APPENDIX: WAVE FUNCTIONS}

The number of $Z_{2}$ even zero modes is equal to three only if $q M=4$ and 5. For simplicity, we set $\tau=i \operatorname{Im} \tau$. Their wave functions are approximated up to the normalization $\mathcal{N}$ as

$$
\begin{aligned}
\Theta_{+}^{0,4}(z) & \sim 1+2 e^{-4 \pi \operatorname{Im} \tau}+\cdots \quad \text { at } z=0 \\
& \sim 1+2 e^{-4 \pi \operatorname{Im} \tau}+\cdots \quad \text { at } z=\frac{1}{2}, \\
& \sim 2\left(e^{-\pi \operatorname{Im} \tau}+e^{-9 \pi \operatorname{Im} \tau} \cdots\right) \quad \text { at } z=\frac{i}{2} \operatorname{Im} \tau, \\
& \sim-2\left(e^{-\pi \operatorname{Im} \tau}+e^{-9 \pi \operatorname{Im} \tau} \cdots\right) \quad \text { at } z=\frac{1}{2}+\frac{i}{2} \operatorname{Im} \tau
\end{aligned}
$$

for $\Theta_{+}^{0,4}(z)$,

$$
\begin{aligned}
\Theta_{+}^{1,4}(z) & \sim \sqrt{2}\left(e^{-(1 / 4) \pi \operatorname{Im} \tau}+e^{-(9 / 4) \pi \operatorname{Im} \tau}+\cdots\right) \text { at } z=0, \\
& \sim \sqrt{2}\left(-e^{-(1 / 4) \pi \operatorname{Im} \tau}-e^{-(9 / 4) \pi \operatorname{Im} \tau}+\cdots\right) \text { at } z=\frac{1}{2}, \\
& \sim \sqrt{2}\left(e^{-(1 / 4) \pi \operatorname{Im} \tau}+e^{-(9 / 4) \pi \operatorname{Im} \tau} \cdots\right) \text { at } z=\frac{i}{2} \operatorname{Im} \tau, \\
& \sim \sqrt{2}\left(e^{-(1 / 4) \pi \operatorname{Im} \tau}+e^{-(9 / 4) \pi \operatorname{Im} \tau} \cdots\right) \text { at } z=\frac{1}{2}+\frac{i}{2} \operatorname{Im} \tau
\end{aligned}
$$

for $\Theta_{+}^{1,4}(z)$,

$$
\begin{aligned}
\Theta_{+}^{2,4}(z) & \sim 2\left(e^{-\pi \operatorname{Im} \tau}+e^{-9 \pi \operatorname{Im} \tau}+\cdots\right) \quad \text { at } z=0, \\
& \sim 2\left(e^{-\pi \operatorname{Im} \tau}+e^{-9 \pi \operatorname{Im} \tau}+\cdots\right) \quad \text { at } z=\frac{1}{2}, \\
& \sim 1+2 e^{-4 \pi \operatorname{Im} \tau}+\cdots \quad \text { at } z=\frac{i}{2} \operatorname{Im} \tau, \\
& \sim-1-2 e^{-4 \pi \operatorname{Im} \tau} \cdots \quad \text { at } z=\frac{1}{2}+\frac{i}{2} \operatorname{Im} \tau
\end{aligned}
$$

for $\Theta_{+}^{2,4}(z)$,

$$
\begin{aligned}
\Theta_{+}^{0,5}(z) & \sim 1+2 e^{-5 \pi \operatorname{Im} \tau}+\cdots \quad \text { at } z=0, \\
& \sim 1-2 e^{-5 \pi \operatorname{Im} \tau}+\cdots \quad \text { at } z=\frac{1}{2}, \\
& \sim 2\left(e^{-(5 / 4) \pi \operatorname{Im} \tau}+e^{-(45 / 4) \pi \operatorname{Im} \tau} \cdots\right) \quad \text { at } z=\frac{i}{2} \operatorname{Im} \tau, \\
& =0 \quad \text { at } z=\frac{1}{2}+\frac{i}{2} \operatorname{Im} \tau
\end{aligned}
$$

for $\Theta_{+}^{0,5}(z)$,

$$
\begin{aligned}
\Theta_{+}^{1,5}(z) & \sim \sqrt{2}\left(e^{-(1 / 5) \pi \operatorname{Im} \tau}+e^{-(16 / 5) \pi \operatorname{Im} \tau}+\cdots\right) \quad \text { at } z=0, \\
& \sim \sqrt{2}\left(-e^{-(1 / 5) \pi \operatorname{Im} \tau}+e^{-(16 / 5) \pi \operatorname{Im} \tau}+\cdots\right) \text { at } z=\frac{1}{2}, \\
& \sim \sqrt{2}\left(e^{-(9 / 20) \pi \operatorname{Im} \tau}+e^{-(49 / 20) \pi \operatorname{Im} \tau} \cdots\right) \text { at } z=\frac{i}{2} \operatorname{Im} \tau, \\
& =0 \quad \text { at } z=\frac{1}{2}+\frac{i}{2} \operatorname{Im} \tau
\end{aligned}
$$

for $\Theta_{+}^{1,5}(z)$, and

$$
\begin{array}{rlrl}
\Theta_{+}^{2,5}(z) & \sim \sqrt{2}\left(e^{-(4 / 5) \pi \operatorname{Im} \tau}+e^{-(9 / 5) \pi \operatorname{Im} \tau}+\cdots\right) & \text { at } z=0 \\
& \sim \sqrt{2}\left(e^{-(4 / 5) \pi \operatorname{Im} \tau}-e^{-(9 / 5) \pi \operatorname{Im} \tau}+\cdots\right) & \text { at } z=\frac{1}{2}, \\
& \sim \sqrt{2}\left(e^{-(1 / 20) \pi \operatorname{Im} \tau}-e^{-(81 / 20) \pi \operatorname{Im} \tau} \cdots\right) & \text { at } z=\frac{i}{2} \operatorname{Im} \tau, \\
& =0 \quad \text { at } z=\frac{1}{2}+\frac{i}{2} \operatorname{Im} \tau
\end{array}
$$

for $\Theta_{+}^{2,5}(z)$. At $z=\frac{1}{2}+\frac{i}{2} \operatorname{Im} \tau$, we have $\Theta_{+}^{0,5}(z)=\Theta_{+}^{1,5}(z)=$ $\Theta_{+}^{2,5}(z)=0$ for $\operatorname{Re} \tau=0$. However, when we set $\operatorname{Re} \tau \neq 0$, we obtain nonvanishing values of $\Theta_{+}^{0,5}(z), \Theta_{+}^{1,5}(z)$, and $\Theta_{+}^{2,5}(z)$ at the fixed point $z=\frac{1}{2}+\frac{\tau}{2}$. 
[1] C. Bachas, arXiv:hep-th/9503030.

[2] M. Berkooz, M. R. Douglas, and R. G. Leigh, Nucl. Phys. B480, 265 (1996).

[3] R. Blumenhagen, L. Goerlich, B. Kors, and D. Lust, J. High Energy Phys. 10 (2000) 006.

[4] C. Angelantonj, I. Antoniadis, E. Dudas, and A. Sagnotti, Phys. Lett. B 489, 223 (2000).

[5] D. Cremades, L. E. Ibanez, and F. Marchesano, J. High Energy Phys. 05 (2004) 079.

[6] H. Abe, K. S. Choi, T. Kobayashi, and H. Ohki, J. High Energy Phys. 06 (2009) 080.

[7] H. Abe, T. Kobayashi, H. Ohki, A. Oikawa, and K. Sumita, Nucl. Phys. B870, 30 (2013).

[8] H. Abe, T. Kobayashi, H. Otsuka, Y. Takano, and T. H. Tatsuishi, Phys. Rev. D 94, 126020 (2016).

[9] H. Abe, T. Kobayashi, and H. Ohki, J. High Energy Phys. 09 (2008) 043.

[10] R. Blumenhagen, M. Cvetic, F. Marchesano, and G. Shiu, J. High Energy Phys. 03 (2005) 050.

[11] T. H. Abe, Y. Fujimoto, T. Kobayashi, T. Miura, K. Nishiwaki, and M. Sakamoto, J. High Energy Phys. 01 (2014) 065; Nucl. Phys. B890, 442 (2014).

[12] T. Kobayashi and S. Nagamoto, Phys. Rev. D 96, 096011 (2017).

[13] H. Abe, K. S. Choi, T. Kobayashi, and H. Ohki, Nucl. Phys. B814, 265 (2009).

[14] H. Abe, T. Kobayashi, K. Sumita, and Y. Tatsuta, Phys. Rev. D 90, 105006 (2014).

[15] T. Kobayashi, Y. Tatsuta, and S. Uemura, Phys. Rev. D 93, 065029 (2016).

[16] Y. Matsumoto and Y. Sakamura, Prog. Theor. Exp. Phys. 2016, 053B06 (2016).
[17] Y. Fujimoto, T. Kobayashi, K. Nishiwaki, M. Sakamoto, and Y. Tatsuta, Phys. Rev. D 94, 035031 (2016).

[18] T. Kobayashi, K. Nishiwaki, and Y. Tatsuta, J. High Energy Phys. 04 (2017) 080.

[19] W. Buchmuller, M. Dierigl, F. Ruehle, and J. Schweizer, Phys. Rev. D 92, 105031 (2015).

[20] W. Buchmuller, M. Dierigl, F. Ruehle, and J. Schweizer, Phys. Lett. B 750, 615 (2015).

[21] W. Buchmuller and J. Schweizer, Phys. Rev. D 95, 075024 (2017).

[22] M. Ishida, K. Nishiwaki, and Y. Tatsuta, Phys. Rev. D 95, 095036 (2017); J. High Energy Phys. 07 (2018) 125.

[23] H. Abe, M. Ishida, and Y. Tatsuta, arXiv:1806.10369.

[24] H. M. Lee, H. P. Nilles, and M. Zucker, Nucl. Phys. B680, 177 (2004).

[25] P. Fayet and J. Iliopoulos, Phys. Lett. 51B, 461 (1974).

[26] D. M. Ghilencea, S. Groot Nibbelink, and H. P. Nilles, Nucl. Phys. B619, 385 (2001).

[27] S. Groot Nibbelink, H. P. Nilles, and M. Olechowski, Phys. Lett. B 536, 270 (2002); Nucl. Phys. B640, 171 (2002).

[28] H. Abe, T. Higaki, and T. Kobayashi, Prog. Theor. Phys. 109, 809 (2003).

[29] T. h. Abe, Y. Fujimoto, T. Kobayashi, T. Miura, K. Nishiwaki, M. Sakamoto, and Y. Tatsuta, Nucl. Phys. B894, 374 (2015).

[30] Z. z. Xing, H. Zhang, and S. Zhou, Phys. Rev. D 77, 113016 (2008).

[31] S. Antusch and V. Maurer, J. High Energy Phys. 11 (2013) 115. 